Relations industrielles

Industrial Relations

\title{
Opposition entre usage et convention écrite
}

\section{Roger Chartier}

Volume 14, numéro 1, janvier 1959

URI : https://id.erudit.org/iderudit/1022343ar

DOI : https://doi.org/10.7202/1022343ar

Aller au sommaire du numéro

\section{Éditeur(s)}

Département des relations industrielles de l'Université Laval

\section{ISSN}

0034-379X (imprimé)

1703-8138 (numérique)

Découvrir la revue

\section{Citer ce document}

Chartier, R. (1959). Opposition entre usage et convention écrite. Relations industrielles / Industrial Relations, 14(1), 92-101.

https://doi.org/10.7202/1022343ar
Résumé de l'article

Quand l'arbitre se trouve en présence d'une clause très claire de la convention collective qui s'oppose à un cas également clair d'usage établi de longue date et admis des deux parties, comment peut-il trancher le litige ?
Tous droits réservés @ Département des relations industrielles de l’Université Laval, 1959
Ce document est protégé par la loi sur le droit d'auteur. L’utilisation des services d'Érudit (y compris la reproduction) est assujettie à sa politique d'utilisation que vous pouvez consulter en ligne.

https://apropos.erudit.org/fr/usagers/politique-dutilisation/ 


\section{Opposition entre usage et convention écrite}

\section{Roger Chartien}

(avec la collaboration de Me Marcel Crête, co-signataire, et la dissidence de M. Ivan Legault)

Quand l'arbitre se trouve en présence d'une clause très claire de la convention collective qui s'oppose à un cas également clair d'usage établi de longue date et admis des deux parties, comment peut-il trancher le litige?

\section{A) Preuve et argumentation des parties}

\section{1-Position du Syndicat}

Depuis 13 ans environ, quelque 14 «millwrights » et soudeurs de la division du Carbure de la Compagnie travaillent d'après une cédule d'opération continue, sept jours par semaine, les uns sur une équipe «barrée» (locked shift) de 4 h. p.m. à minuit, et les autres sur une équipe du même genre, de minuit à 8 h. a.m.

(L'entretien s'effectue par les soins des ouvriers de jour, du lundi au vendredi. Sauf urgence, aucun employé ne travaille sur l'équipe de jour le samedi, le dimanche et les jours de fête.)

Les employés qui ont logé ce grief ont toujours été payés temps et demi pour le travail du dimanche et des fêtes, mais sans recevoir, ces jours, ni la prime de travail du dimanche, ni la prime d'équipe, ni la prime d'équipe «barrée ». Les seuls ajustements, dans ce domaine, ont été faits lors de la réduction de la semaine de travail de 48 à 44,42 , puis 40 heures.

D'après les articles 503 et 504 de la convention collective, les heures de travail en général, et particulièrement les horaires (cédules) de travail des équipes, ne pourront se modifier que de l'accord des parties: «cédules agréées par les deux parties * (503), et « à la discrétion de la gérance et avec le consentement du syndicat 》 (504).

En conséquence, la Compagnie demande, en octobre 1956, le consentement du Syndicat pour distribuer les employés en cause selon une cédule de trois équipes rotatives, ce qui supprimerait le temps et demi les dimanches et jours de fête, s'y substituant la prime d'équipe, la prime d'équipe «barrée » et la prime du travail dominical, toutes trois ensemble moins rémunératrices que le temps et demi.

Le Syndicat opposant son refus, l'ancienne cédule est maintenue. Mais le 19 avril 1957, la Compagnie, de sa propre initiative et sans consulter le Syndicat, supprime le temps et demi du dimanche, accordant en son lieu les trois primes précitées. Et il en va ainsi depuis lors.

Le mémoire syndical demande donc à ce conseil d'arbitrage de décider que la Compagnie a indîment supprimé le paiement aux employés concernés du temps et demi le dimanche et les fêtes; et que les intéressés seront remboursés des pertes de salaire ainsi subies depuis le 19 avril 1957 inclusivement. 
Le procureur syndical, dans son angumentation, souligne qu'il s'agit ici d'un pur cas d'usage prépondérant («past practices») qui doit emporter l'adhésion des arbitres. Sous de nombreuses conventions collectives passées, et même sous la présente (depuis sa signature en août 1956 jusqu'au 19 avril 1957), les employés en question ont toujours reçu temps et demi pour leur travail du dimanche et des fêtes. Il ne peut donc s'agir d'une «erreur » de la part de la Compagnie! Et même si on la supposait, comment expliquer que, découverte en octobre 1956, elle n'ait été «corrigée » que six mois plus tard?

Il y avait décidément entre les parties accord implicite pour accorder, dans ces cas, le temps et demi, accord qu'on ne peut mettre au rancart unilatéralement. Enfin, de dire le procureur syndical, les travailleurs en cause s'attendaient à recevoir régulièrement le temps et demi, et se trouvent donc lésés en son défaut; et les employés de l'entretient n'auraient jamais accepté la cédule sans le temps et demi.

\section{Position de LA Compagnie}

La Compagnie admet tous les éléments de la preuve syndicale. En argumentation, elle rappelle qu'il ne s'agit pas ici d'une modification unilatérale de cédule: elle avait souhaité et proposé cette modification, mais faute de consentement du Syndicat (nécessaire d'après les articles 503 et 504 de la convention collective), elle s'en était tenue à l'ancienne cédule, de l'aveu même du Syndicat. Ce qui est en cause, c'est une somme d'argent en plus ou en moins à recevoir (temps et demi ou trois primes). Et la Compagnie, par son geste, entend non pas violer, mais corriger une violation de la convention collective.

Les 14 travailleurs en cause, de continuer le procureur patronal, sont des travailleurs de relève i «shift workers »), et c'est l'article 509 de la convention qui s'applique à leur cas. Cet article est ainsi conçu:

«Les travailleurs de relève de l'opération et de l'entretien qui sont normalement cédulés pour travailler le dimanche recevront une prime de dix (10) cents l'heure pour tout travail fait le dimanche et les jours de fête cédulés dans cette convention.

«Cette prime ne s'applique pas à tout employé qui recevrait normalement temps-et-demi durant ces jours. »

A tort, les «millwrights » et soudeurs en question avaient été rémunérés selon la première phrase de l'article 508, la Compagnie omettant «par erreur» de tenir compte de la seconde phrase de cet article, dont le texte intégral se présente comme suit:

\& Le taux de temps-et-demi sera payé pour tout travail fait le dimanche ou un jour de fête. Ceci ne s'applique pas aux travailleurs régis par le paragraphe 509. »

La Compagnie a certes le droit de corriger son erreur et de revenir aux stipulations fort claires de la convention. Et ce faisant, elle ne peut donner prise à aucun grief fondé. 


\section{B) COMMentaires des arbitres}

Nous n'accordons pas plus de poids qu'il faut à l'argument patronal de «l'erreur corrigée»; selon nous, le geste de la Compagnie est tout simplement la manifestation de son indisposition devant le refus du Syndicat de consentir au ohangement de cédule par elle proposé. Mais ce qu'il importe de savoir, c'est si cette forme de mauvaise humeur est légale. Et ce conseil d'arbitrage entend étudier le problème en son fond même.

\section{1-La nature exacte du litige}

\section{a) La convention collective}

La prétention patronale suivant laquelle les 14 travailleurs en cause sont des « shift workers (travailleurs de relève) est admise par le mémoire syndical, qui indique clairement que ces employés travaillent sur deux \&locked shifts 》 et que leur horaire normal inclut le travail dominical («cédule d'opération continue, sept jours par semaine », de pnéciser le mémoire).

( D'autre part, conme les deux parties reconnaissent qu'il ne s'agit pas d'une modification d'horaire (même s'il est en preuve que la Compagnie l'eut souhaitée), il n'a pu y avoir violation des articles 503 et 504 de la convention par changement unilatéral de cédule.)

Sur le plan strict de la convention, donc, deux articles s'appliquent clairement et sans le moindre doute: ce sont les articles (précités) 508 (dernière phrase) et surtout 509, qui accorde aux travailleurs de relève normalement cédulés pour travailler le dimanche une prime de dix (10) cents l'heure pour tout travail fait le dimanche et les jours de fête cédulée \$, en plus évidemment des surprimes d'équipe et d'équipe «barrée » prévues ailleurs dans la convention.

\section{b) L'usage}

Mais le Syndicat se situe sur un plan tout différent: à un texte contractuel parfaitement limpide qui, considéré seul, obligerait les arbitres à renvoyer immédiatement son grief, il oppose un usage («past practices $\gg$ ) constant, de longue date (13 ans), indubitable et d'ailleurs admis par la Compagnie. Jusqu'au 19 avril 1957, en effet, sous la présente convention comme sous plusieurs conventions antérieures, les travailleurs en question ont toujours été payés temps et demi pour le travail du dimanche et des fêtes (mais sans recevoir les trois primes - travail dominical, équipe et équipe «barrée 》 - bien entendu).

Le différend se trouve donc clairement défini: c'est un pur cas de convention collective qui s'oppose à un pur cas d'usage bien établi. Et pour compliquer davantage le problème, la Compagnie, pour sa part, prétend avoir le droit de mettre fin unilatéralement à un accond verbal, même de longue date, si ce faisant elle ne viole pas la convention. Le Syndicat, de son côté, souligne que les travailleurs en cause avaient droit à s'attendre au temps et demi, qu'ils se trouvent lésés de ne le point recevoir, et enfin qu'ils n'auraint pas accepté la présente oédule sans le temps et demi. 
Comment des arbitres peuvent-ils trancher un différend de ce genne? Qu'advient-il quand un texte clair d'une convention écrite s'oppose à un usage reconnu de tous?

Les relations humaines, dans le monde industriel comme ailleurs, se modèlent en partie sur le passé, ce qui explique l'influence profonde des usages sur le règlement des conflits de travail. Mais l'attitude des parties à l'égard de l'argur ment de l'usage (surtout quand celui-ci s'oppose à une entente écrite) variera le plus souvent selon les besoins de la cause: dans un cas, par exemple, le Syndicat (ou la Compagnie) pourra défendre avec vigueur et même avec férocité \&la convention collective, loi sacrée des parties, fruit de luttes patientes et douloureuses, signe de démocratie industrielle et symbole d'une émancipation graduelle et efficace », alors que dans le cas suivant le même syndicat (ou la même Compagnie) invoquera justice, bon sens, sain réalisme et large ouverture de compas pour inviter les arbitres à «délaisser les froids ossements d'une convention collective imparfaite, ébauchée à la hâte, rigide et immobiliste pour trancher en pleine viande de la relation totale des parties, et plus précisément des usages non écrits 》.

Le problème, c'est qu'on exige des arbitres, en cette matière, plus d'esprit de suite, de logique et de conséquence, et moins de «flexibilité ». Ces derniers peuvent quand même s'aider de l'étude attentive des anguments de deux «écoles de pensée 》 dont les tenants ont souvent comme caractère dominant de passer de l'une à l'autre selon les besoins du moment.

\section{2-Deux accents différents sur l'usage et la convention écrite}

\section{a) Le caractère «sacré » de la convention collective}

Le juriste Paul Durand, auteur d'un puissant Traité de droit du travail (Paris: Librairie Dalloz, 1956, tome III) et partisan tenace du particularisme du droit du travail en France, tout en rendant compte des libertés nombreuses prises par les juridictions arbitrales à l'égard du droit commun, exprime quand même des idées assez sobres sur la pratique, dangereuse selon lui, de donner préséance à des usages non écrits et à des «fantaisies individuelles 》 sur des stipulations précises d'une convention écrite. Selon lui, la convention collective et les coutumes (formes plus stables et plus communément observées des usages), toutes deux formées par et dans le milieu professionnel, ne sont cependant pas d'égale valeur aux yeux d'un arbitre. La convention, en effet, présente comme source de droit une évidente supériorité sur la coutume, car elle constitue l'oeuvre particulièrement réfléchie, consciente et libre des organes chargés de réglementer la vie professionnelle. De plus, elle est écrite, généralement plus complète et plus précise, et plus facile d'accès et d'évaluation que les coutumes.

Donc, estiment les tenants de cette doctrine, la convention écrite, même mal conçue, fautivement rédigée ou imparfaite, a énormément plus de poids que toute entente verbale, fût-elle établie de longue date. Les clauses d'une convention collective ont un caractère sacré, car elles sont la loi des parties. Il peut être dangereux d'opposer toujours lettre et esprit, d'injecter constamment dans la convention collective des «fantaisies individuelles》 ou des «idéologies sociales 》. Les arbitres ne doivent ni la modifier, ni y ajouter, ni surtout la réduire à un rôle 
subalterne. Mieux vaut la laisser s'expliquer d'elle-même, et éviter de toujours invoquer une quelconque «intention des parties lors de la signature 》 pour la contrecarrer. Sans quoi cette convention finit par ne plus rien signifier pour les. deux parties, chacune des parties pouvant y mettre la hache et utiliser des tiersarbitres pour en provoquer l'effritement.

\section{b) L'extension du champ des relations arbitrales}

L'autre « école», plus large, recule les limites des relations qui unissent les parties. Ainsi que s'exprime le professeur Chamberlain:

\& Even when the argreement has been signed and sealed, it does not encompass the whole of the relationship and an arbitrator would be in error so to assume. It sets out the explicit understandings of the parties, but there is usually mone existing between them than is made explicit. The relationship as well as the agreement creates obligations.

(Jean T. McKelvey, ed. Management Rights and the Arbitration Process. Washington: BNA Incorp., 1956, p. 143.)

C'est une autre façon de dire que les relations des parties ne se limitent pas au contenu de la convention collective; qu'il existe entre elles une multitude d'accords à durée plus ou moins longue qui contribuent à la flexibilité et à l'harmonie des relations industrielles, et sans lesquels le dynamisme si nécessaire à ces relations serait dangereusement ralenti ou ferait complètement défaut.

Le professeur Benjamin Werne, résumant une décision arbitrale rendue aux Etats-Unis dans le cas de Coca-Cola Bottling Co. (9, LA 197), exprime ce point de vue avec encore plus de clarté:

(A union-management contract is far more than words on paper, it is also all the oral understandings, interpretations and mutually acceptable habits of action which have grown up around it over the course of time. Stable and peaceful relations between the parties depend upon the development of a mutually satisfactory superstructure of understanding which gives operating significance and practically to the purely legal wording of the written contract. Peaceful relations depend, further, upon both parties faithfully living up to their mutual commitments as embodied not only in the actual contract itself but also in the modes of action which have become an integral part of it.

«If any of these... becomes undesirable to either party, it should obtain the consent of the other party to revise the contract, in this langer sense, accordingly. The terms of a contract cannot be unilaterally changed during the period which it covers....

(The Law of Labor Relations. New York: Macmillan, 1951, p. 328.)

Mais le professeur Chamberlain, si bien lancé, met les freins:

«I suspect that there are some kinds of practices which do create obligations on management, though I would find it difficult to spell out the nature of such practices or the criteria by which they may be identified, or even the nature of management's obligations under them. I suspect they are quite limited in number....

(Op. cit., p. 147.) 
«In examining the meaning of an agreement, it is proper to inquire about the conditions under which the bargain took place with a presumption that the normal practices which did exist are expected to continue except as the agreement would require or justify alteration and except as conditions make such past circumstances no longer feasible of Appropriate. Both parties have rights to stability and protection from unbargained changes ...

(Op. cit., p. 120.)

\section{3-Ia position de ce conseil d'arbitrage}

Nous estimons que la formulation d'après laquelle la convention collective ne constitue qu'une partie des relations entre syndicat et direction est très réaliste, très exacte et très pertinente pour des arbitres. C'est vrai qu'il existe entre les parties toute une série d'accords oraux qui, si leur existence est péremptoirement établie devant un conseil d'arbitrage comme usages, et non pas seulement comme pratiques transitoires, cessent d'être assimilables à des contrats synallagmatiques à durée indéterminée, i.e., ne sont plus réalisables unilatéralement au gré de l'une ou l'autre des parties. Ce ne sont plus là des précédents mouvants, épars, flexibles et explicables par les «erreurs》 ou les «concessions》 de l'une des parties, mais de véritables obligations contractuelles qui lient les deux parties.

Ce conseil d'arbitrage croit aussi fermement en la valeur et en l'importance, pour la paix industrielle, de la convention collective, et estime qu'il est de son devoir d'en assurer le respect dans toute la mesure possible, indiquant souvent aux parties, quoique d'une façon indirecte, l'utilité et la façon de l'améliorer en négociations directes pour en faire un instrument à la fois plus souple et plus explicite. Cette convention, dont l'une ou l'autre des parties ferait si facilement des choux et des raves à un moment donné au nom de «past practices 》 qui la contrecarrent, elle est quand même l'expression la plus claire, la plus sûre et la plus solennelle de l'accord des parties à un stade donné de l'évolution de leurs relations. A ce titre, elle mérite que les arbitres... et les parties elles-mêmes la prennent au sérieux.

Si donc la convention collective est muette ou plutôt ambiguë sur un point qui d'autre part fait l'objet d'un usage clairement démontré, nous posons comme premier principe qu'un conseil d'arbitrage doit respecter cet usage comme s'il était inscrit dans une convention collective écrite, et contraindre au besoin les deux parties à n'y pas toucher durant la durée de la convention en cours.

Si d'autre part on oppose un usage mal démontré (et qui est plutôt une pratique sporadique) à un texte de convention peu clair ou fort clair, c'est la convention qui évidemment, l'emportera dans le jugement des arbitres.

Si enfin on met en opposition un usage mal prouvé et un texte de convention ambigu, ou encore un usage parfaitement démontré et une stipulation écrite parfaitement limpide, c'est la convention écrite qui l'emportera aux yeux des arbitres, à cause du caractère plus sûr, plus précis et plus solennel de cette dernière.

Quand des conflits comme celui qui est présentement devant nous viennent à s'élever, c'est signe qu'il est sans doute opportun pour les parties de sacrifier quelque peu de flexibilité et de latitude sur un point donné pour insérer un usage de longue date dans leur convention collective. 
Et quand l'une des parties (la Compagnie, en l'occurrence) prend l'initiative de la rupture d'une entente verbale fort claire, communément admise et respectée depuis de nombreuses années en profitant d'une faille de la convention collective, elle pourra gagner légalement son point devant un conseil d'arbitrage, mais elle s'exposera à de sérieuses représailles, dans la même veine, de la part de l'autre partie qui, au nom des mêmes principes, gagnera vraisemblablement elle aussi son point de droit devant d'autres arbitres. Mais le présent conseil d'arbitrage estime que c'est là une façon plutôt originale et fort risquée de promouvoír au sein d'une entreprise l'harmonie industrielle.

Eu égard aux principes exposés précédemment, ce conseil d'arbitrage, donnant priorité à une stipulation écrite très précise de la convention collective sur un usage fort clair en sens contraire, DÉCIDE À L'UNANIMITÉ, l'arbitre syndical étant dissident, que la Compagnie ne peut être contrainte à payer les employés en cause au taux de temps et demi, et donc que le grief du Syndicat doit être renvoyé.

Le président de ce conseil d'arbitrage, conscient de son devoir, recommande instamment à la Compagnie, sans l'y contraindre, de se rendre à la demande syndicale et de payer aux employés concernés, selon une coutume établie de longue date, le temps et demi moins les trois primes, depuis le 19 avril 1957 inclusivement jusqu'au moment où, en négociations directes, elle aura réglé ce problème avec le Syndicat.

\section{Dissidence de M. Ivan-A. Legault, arbitre syndical}

Sous réserve de notre dissidence sur la considération principale et la décision qu'elle fait suivre, nous déclarons partager plusieurs des points de vue de nos collègues; points de vue que l'on retrouve dans le volumineux texte de la décision afférante au présent grief.

Nous sommes d'accord avec nos collègues notamment sur le fait qu'il ne doit pas être accordé «plus de poids qu'il faut à l'argument patronal de \& l'erreur corrigée »; (et que) selon nous, le geste de la Compagnie est tout simplement la manifestation de son indisposition devant le refus du Syndicat de consentir au changement de cédule par elle proposé ».

Nous sommes aussi d'accord avec la majorité des membres de ce Conseil lorsqu'ils disent:

Nous estimons que la formulation d'après laquelle la convention collective ne constitue qu'une partie des relations entre syndicat et direction est très réaliste, très exacte et très pertinente pour des arbitres. C'est vrai qu'il existe entre les parties toute une série d'accords oraux qui, si leur existence est péremptoirement établie devant un Conseil d'arbitrage comme usages, et non pas seulement, comme pratiques transitoires, cessent d'être assimilables à des contrats symallagnatiques, à durée indéterminée, i.e., ne sont plus résiliables unilatéralement au gré de l'une ou l'autre des parties. Ce ne sont plus là des précédents mouvants, épars flexibles iet explicables par les «enreurs 》 où les «concessions》 de l'une des parties, mais de véritables obligations contractuelles qui lient les deux parties.

Nous avons par ailleurs inscrit notre dissidence sur l'opinion majoritaire voulant que «ce qu'il importe de savoir, c'est si cette forme de mauvaise humeur 
de la Compagnie est légale », et nos collègues de conclure plus loin que: «si l'on met en opposition (...) un usage parfaitement démontré et une stipulation écrite parfaitement limpide, c'est la convention écrite qui l'emportera aux yeux des arbitres, à cause du caractère plus sûr, plus précis et plus solennel de cette dernière »; et de là, la majorité du Conseil de décider «que la Compagnie ne peut être contrainte à payer les employés en cause au taux de temps et demi, et donc que le grief du syndicat doit être renvoyé ».

Nos collègues identifient donc ici le différend, comme étant un pur cas de stipulations écrites opposé à un pur cas d'usage bien établi.

Nous croyons pour notre part que les faits déposés devant ce Conseil exigent d'être examinés dans une autre optique et réclament une conclusion différente de la décision majoritaire.

Selon notre opinion, le problème se situe plutôt en termes de droit à des avantages acquis par l'usage, qu'à savoir si les termes de la convention de travail prévalent sur l'\&usage » ou vice-versa.

《Avantages acquis» et «usage», ce sont là deux termes que l'on retrouve fréquemment en relation mais qui n'en sont pas synonymes pour autant.

Pour ce qui est de l'«usage» que nous assimilons aux «past practices》, Durand, auteur auquel il a déjà été référé, en fonde l'existence sur les critères suivants:-

《-la généralité, (la gratification n'était pas versée à un ou plusieurs salariés déterminés, mais à l'ensemble du personnel ou de la catégorie professionnelle considérée).

-la constance (qui exclut l'éventualité d'un acte discrétionnaire de l'employeur).

-la fixité (révélant que l'employeur n'était pas libre de fixer à son gré le taux de la gratification, mais qu'il se conformait à un usage).» (Cf. ibid. T. II, no 363 , p. 687.)

La question de l'\&usage» ne soulève cependant pas de problème dans le cas qui nous est soumis; le Conseil d'arbitrage ayant reconnu et confirmé l'«usage » d'après les dépositions non contradictoires des parties, dépositions correspondant en tout aux critères signalés plus haut.

Quant à la règle de la conservation des «avantages acquis », pour Durand (op. cit., T. III, pp. 510, 515), elle ne laisse aucun doute en dépit du fait que la portée de ce principe soulève d'assez grandes difficultés. D'après le même auteur:

1-L'《avantage » ne correspond pas à une notion générale, définie par le Droit du Travail. Dans le droit des conventions collectives, l'avantage désigne le traitement plus favorable dont les salariés peuvent déjà bénéficier. Il peut consister i.e. dans une rémunération plus élevée (taux de salaire supérieur, complément de salaire, (...) etc.)

2-Un avantage n'est acquis que si l'employeur est obligé de le consentir. Peu importe, dit-il, que cette obligation résulte d'un 
contrat individuel de travail, d'un règlement intérieur, d'《usages », d'une convention collective, d'une sentence arbitrale, ou d'un texte légal ou règlementaire (...).

3-Un avantage ne peut être considéné comme acquis que s'il est susceptible de se renouveler dans le temps (...). Il suffit, ajoute-til que la règle prévoyant l'avantage ait un caractère permanent et qu'elle soit susceptible d'autres applications dans l'avenir.

Or, après avoir examiné la déposition des parties (cf. décision majoritaire, pp. 50-51-52), à la lumière de ces principes, nous considérons, d'une part, que le temps et demi payé aux «millwrights» pour le travail du dimanche faisait partie des «avantages acquis » à cette catégorie d'employés, et, d'autre part, que si la convention collective de travail, par les articles 508-509, était susceptible de mettre fin à un «usage » établi jusqu'à la mise en vigueur de la convention, celleci n'a pas entériné pour autant l'abolition des «avantages acquis 》 à ces «millwrights 》 dont le «contrat 》 était en cours à la date d'entrée en vigueur de cette convention. Nous constatons, au contraire, que la convention reste muette à cet égard: elle n'indique pas de façon positive l'intention des parties de mettre fin à la fois aux «avantages aoquis » et à l'«usage ».

A notre avis, il y a présomption justifiée à l'endroit des plaignants. N'a-t-il pas, en effet, été clairement établi que les parties s'étaient entendues au cours des négociations préalables à la signature de la convention de travail à l'effet que l'avantage en question serait maintenu? En eût-il été autrement, pourquoi la Compagnie aurait-elle entamé des négociations postérieusement à la signature de la Convention et tenté d'opérer un virement en ce qu'elle-même du fait de son initiative, considérait être une obligation? Ne semble-t-il pas étrange que, n'étant parvenue à ses fins, la Compagnie ait, par la suite, attendu six mois après sa supposée erreur pour convertir et réduire le paiement du temps et demi au versement des « primes»?

C'est ainsi que la décision majoritaire, vue dans ces perspectives, équivaut dans son verdict à déclarer que le syndicat et les travailleurs concernés, avec la signature de la nouvelle convention, avaient renoncé à l'avantage du temps et demi dont ils jouissaient auparavant; ce qui, à notre avis, est invraisemblable et n'est conforme ni à la preuve déposée ni aux principes gouvernant cette matière.

Citons à notre appui un extrait de la décision arbitrale majoritaire rendue en date du 2 novembre 1948 au sujet du différend survenu entre STANDard Paper Box Ltd et L'Association des employés de Standard Paper Box (Cf. Bulletin no 307 du Ministère du Travail de la Province de Québec), décision dans laquelle MM. Roger Brossard et Jean Delâge invoquaient, dans les termes suivants, les principes dont nous nous réclamons:-

«Il est de principe constant - disaient-ils - que la renonciation à un droit ne se présume pas et qu'elle doit être clairement exprimée, sauf dans le cas où le droit n'est pas exercé pendant une certaine durée, ce qui donne lieu à la prescription. »

C'est pourquoi, en conclusion, après examen de la preuve soumise, nous appuyant sur la jurisprudence citée plus haut, nous soumettons l'opinion minoritaire à l'effet que le temps et demi payé aux \& millwrights » pour le travail du 
dimanche, constitue et fait encore incontestablement partie des «avantages acquis 》 à ce groupe de travailleurs; avantages créés par l'«usage » et reconnus comme tels tant au moment des négociations que lors de la signature de la Convention et en conséquence que le présent Conseil d'arbitrage aurait dû faire droit à la réclamation des ouvriers concernés.

\title{
L'interprétation arbitrale de la convention collective
}

\section{Me Marie-Louis Beauliteu}

\begin{abstract}
Dans un cas de grief récent soumis à la législation fédérale du travail et entendu d Québec, l'arbitre patronal a proposé une définition étroitement restrictive de l'sinterprétation d'une clause de convention collective », prétendant que des arbitres chargés d'interpréter une telle clause ne peuvent dire ni qui est couvert par elle, ni de quelle façon. Me Beaulieu, président du conseil d'arbitrage, donne ici son avis sur cette question.
\end{abstract}

L'arbitre patronal, à la fin du délibéné, a adopté la position suivante: le tribural a mandat d'interpréter la clause 17 de la convention collective et il ne peut rien faire de plus. Cela est exact et nous sommes parfaitement d'accord avec l'arbitre patrnnal. Mais où nous différons d'opinion avec hi, c'est sur ce qu'il faut entendre par «interpréter une clause de convention collective ». D'après lui, cela signifie donner une opinion, qu'on qualifie de purement théoriqur. En d'autres termes, il prétend - nous croyons synthétiser ici sa pensée - que des arbitres chargés d'interpréter une clause de convention collective ne peuvent dire ni qui est couvert par la clause, ni de quelle façon. Pour nous, cette façon de voir est erronée. Elle est contredite par la doctrine, elle est contre la nature des choses et va à l'encontre de la pratique généralement suivie. Comment peut-on interpréter un texte de convention collective sans traduire le sens des mots dans le concret, ce qui revient à dire, dans la pratique de l'entreprise? $\mathrm{Ce}$ que nous venons d'écrire s'applique aux sentences, sur un conflit d'intérêts, c'est-à-dire quand la décision n'est qu'une simple recommandation, mais s'applique davantage quand la décision lie les parties, comme dans le cas qui nous occupe, en vertu de l'article 19 du Code National du Travail, qui nous régit dans le moment. Comment les parties pourraient-elles appliquer une décision, car il faut qu'elles l'appliquent puisqu'elle les lie, sans que cette application donne lieu à des difficultés, à des conflits entre l'employeur et ses employés, si la décision ne disait qui elle couvre et jusqu'à quel point? On ne peut vraiment interpréter une loi ou un contrat sans dire de quelle façon ils s'appliquent, et il faut raisonner de la même chose dans l'interprétation des clauses de convention collective.

Vu ce qui précède, la présent tribunal d'arbitrage doit dire [ qui la ] sentence couvre et si elle doit être rétroactive... 